\title{
3D Morphology Distribution Characteristics and Discrete Element Simulation of Sand-Gravel Mixtures
}

\author{
Wu Qi, ${ }^{1}$ Sun Suyu, ${ }^{1}$ Gao Guangliang, ${ }^{2}$ Fang Yi $\mathbb{D}^{1,3}$ and Chen Guoxing ${ }^{1}$ \\ ${ }^{1}$ Institute of Geotechnical Engineering, Nanjing Tech University, Nanjing 210009, China \\ ${ }^{2}$ BGI Engineering Consultants LTD., Beijing 100038, China \\ ${ }^{3}$ National Institute of Natural Hazards, Ministry of Emergency Management of China, Beijing 100085, China
}

Correspondence should be addressed to Fang Yi; yifang@ninhm.ac.cn

Received 19 September 2021; Accepted 5 November 2021; Published 22 November 2021

Academic Editor: Haojie Lian

Copyright (C) $2021 \mathrm{Wu}$ Qi et al. This is an open access article distributed under the Creative Commons Attribution License, which permits unrestricted use, distribution, and reproduction in any medium, provided the original work is properly cited.

Sand-gravel mixtures are typical binary materials, exhibiting highly heterogeneous, discontinuous, and significant structural effects. The contact state between sand and gravel particles has a significant influence on the mechanical properties of the mixtures. This article focused on the complex internal structure and its mesostructural behavior of the mixtures, and a systematic statistical analysis was carried out to study the shape, size, and angularity of the coarse particles. The three-dimensional (3D) shapes of coarse aggregates were approximated to be hexahedron, pentahedron, and tetrahedron. An indicator called angularity and surface texture (AT) index was developed to characterize the combined effect of the coarse aggregate angularity and surface texture. Based on the screening testing and digital image processing, the particle size and AT index of aggregates were extracted, and their means, standard deviations, and statistical distributions were studied. An algorithm for generating 3D aggregates was developed based on the statistical results of the coarse aggregate 3D morphology. The coarse aggregate generating code was written using the fish language in PFC3D. The numerical model was then applied to conduct three typical monotonic or cyclic triaxial test simulations. Retrospective simulation of the laboratory tests using the proposed model showed good agreement, and the reliability of the model is effectively verified. The results interpreted well the mechanism of particle motion and the distribution of interparticle contact force during shearing from mesoscale of the mixtures, which can give better understanding and modeling of the nonlinear behavior of the sand-gravel mixtures.

\section{Introduction}

Hydraulic fracturing is a promising method for increasing the production of oil and gas wells. Hydraulic fracturing is currently the main form of natural gas extraction. It requires a large amount of water mixed with chemicals to be poured into the shale layer for hydraulic fracturing to release natural gas. At present, most of the world's natural gas and oil wells are drilled by hydraulic fracturing [1-4]. Due to the characteristics of deep underground drilling operation of hydraulic fracturing, it often crosses undesirable engineering geology such as a sandy gravel mixture layer $[5,6]$. It is of great significance to guide the construction of engineering to establish a set of fast and effective methods to analyze the mechanical behavior of sandy gravel mixtures.
Sand-gravel mixtures are a special engineering geological material between the soil and the fractured rock; the sandgravel mixture structure presents the mesostructure between sand particles and gravel particles [7]. The contact state and particle morphology of the mixtures have a significant contribution to their mechanical properties [8-12]. If the heterogeneous and discontinuous structural characteristics of the sandy gravel mixtures are ignored and the particle morphology and particle distribution are not considered, the authenticity of the mesostructure characteristic simulation of the material will be reduced, and it is difficult to describe the microscopic mechanical behavior between the particles [13].

Kennedy and Lin [14] constructed polygons to approximate the shape of particles and found that the number of 
sides of the polygon is proportional to the circumference and inversely proportional to the length of the sides. This relationship is linear in double logarithmic coordinates, and the slope indicates the irregularity of the particles. Bowman et al. [15] use the Fourier transform and analysis theory to describe the particle morphology of sand. According to the research of Sukumaran and Ashmawy [16], the surface texture reflects the fine morphology of the particle surface and can be used to describe the roughness of the particle surface and the angular microscale. Cho et al. [17] described the particle morphology based on three aspects: sphericity, edge angle, and roughness. Ferellec and Mcdowell [18] replaced the average radius of curvature of the corners with the average radius of all inscribed circles on the projection contour of the particles, which improved the reproducibility of this parameter.

With the development of computer technology and the emergence of the discrete element method, numerical simulation has gradually become an important method to analyze the particle shape; the elastoplastic finite element method (FEM) was introduced in the initial stage. However, due to the discontinuity of sand, FEM based on continuous medium theory cannot reflect the influence of material parameters such as particle size and particle morphology on the simulation results $[19,20]$. Therefore, the discrete element method (DEM) based on discontinuous medium mechanics has been widely used in the numerical simulation of granular media [21]. This method is low in cost and easy to operate and is very suitable for numerical simulation of discrete media such as sand and gravel.

Mcdowell and Harireche [22] found that adjusting the percentage of pellets empirically reflects the size effect of individual pellet strength. For the particle cluster model, when the particle interaction force exceeds the connection strength, the small particles are detached, which can simulate local or whole fracture. Cheng et al. [23] conducted a simulation of the particle flow of crushable soil, in which the crushed particles used a cluster model of particles formed by the bonding of several small particles, and the randomness of single particle strength was reflected by randomly deducting a certain percentage of small balls. Deluzarche and Cambou [24] used the discrete element code PFC2D and considered breakable clusters of 2D balls. The different parameters were determined from experimental data obtained from laboratory tests performed on rock blocks. The model was validated by comparing the results of the simulation of shearing tests with actual triaxial tests on rockfill material published in the literature. Alaei and Mahboubi [25] focused on numerical modelling of rockfill material with the discrete element method (DEM). Because the DEM models the interaction of separate elements, it was capable of modelling discrete structures of granular materials and particle breakage. Alonso et al. [26] established a distinct element method model developed in which grains were characterized in the clump model. The resulting particle shape approaches real geometries and allows a reasonable breakage evolution, and the particle breakage criterion involves the subcritical propagation of fissures in the grain.
In this study, taking the sand-gravel mixtures distributed at the project site of Binjiang Subway Station of Metro Line 3 in Chengdu as an example, using discrete element numerical simulation and digital image processing technology, the 3D form and distribution of gravel particles are systematically analyzed. Based on the PFC3D particle flow software, a 3D discrete element simulation method for sand-gravel mixtures was proposed. The triaxial numerical test and actual triaxial tests were compared to verify the accuracy of the method and discuss the mesomechanical behavior of the sand-gravel mixtures.

\section{Basic Physical Properties of Sand- Gravel Mixtures}

The sand-gravel mixture samples were taken from Binjiang Subway Station of Metro Line 3, Chengdu. The soil layers in this site from top to bottom are Quaternary Holocene $\left(\mathrm{Q}_{\mathrm{h}}\right)$ artificial fill, alluvial silt, sand and pebble (medium sand lenticel sandwiched in some sections), Quaternary Pleistocene $\left(\mathrm{Q}_{\mathrm{p}}\right)$ alluvial pebbles, and Upper Cretaceous $\left(\mathrm{K}_{2}\right)$ Guankou Formation mudstone. Three groups of sand-gravel mixtures at different depths are used for the particle-size analysis test, and the gradation curve is shown in Figure 1. According to the Unified Soil Classification System (ASTM D2487 [27]), the sand-gravel mixtures belong to poorly graded gravel (GP) with the minimum or maximum dry density of 1.437 or $1.740 \mathrm{~g} / \mathrm{cm}^{3}$, the specific gravity is 2.68 , mean particle size $d_{50}=6.31 \mathrm{~mm}$, coefficient of uniformity $C_{\mathrm{u}}=34.5$, and coefficient of curvature $C_{\mathrm{c}}=0.37$.

\section{The 3D Morphological Characteristics of Sand-Gravel Mixtures}

3.1. Test Materials and Test Methods. Gravel particle morphology is an important parameter of mesophysical and mechanical properties of the sand-gravel mixtures. 110 particles with the diameter range from 50 to $20 \mathrm{~mm}, 117$ particles with the diameter from 20 to $10 \mathrm{~mm}$, and 128 particles with the diameter from 10 to $5 \mathrm{~mm}$ were selected as the research objects. The $3 \mathrm{D}$ shape of gravel particles was captured by digital image processing technology. In order to distinguish the aggregate from the image background more accurately, the background was set to white, and the gravel particles were blackened with ink, as shown in Figure 2.

3.2. 3D Morphological Evaluation Parameters of Particles. The important size parameters of gravel particles include the length of the long axis, the length of the central axis, and the length of the short axis. In order to measure the size of the particles accurately, first, the particles should be neatly arranged on the white paper and a high-definition image was taken. Then, the particles were tiled in situ at a different angle and photographed again. The detailed steps are as follows: 


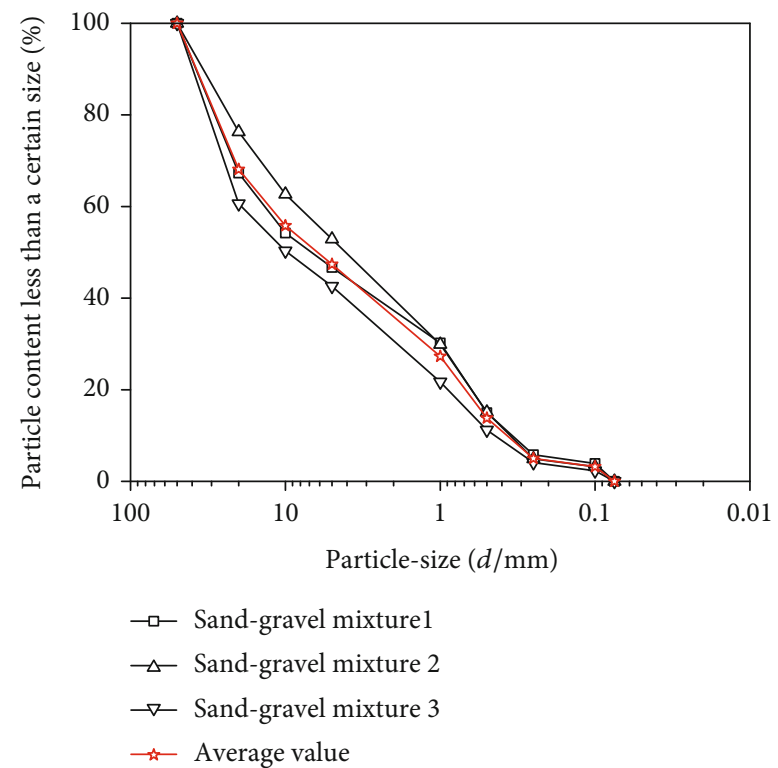

FIGURE 1: Gradation curve of sandy gravel.

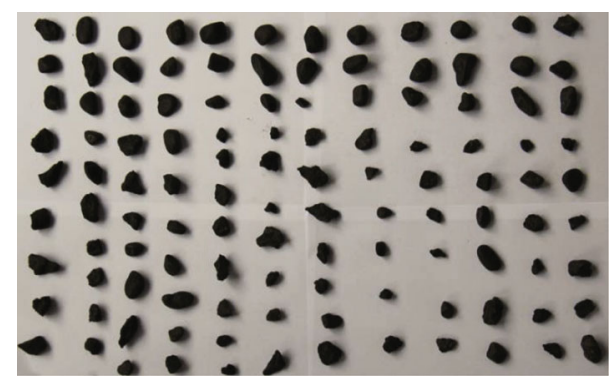

FIGURE 2: Image of blackened gravel particles with the diameter of $5 \sim 50 \mathrm{~mm}$.

(1) Select gravel particle samples of all kinds of particle sizes

(2) Soak gravel particle samples with a certain proportion of ink and put them into the oven for full drying

(3) Arrange gravel particle samples on the white paper neatly, and use a digital camera to take highdefinition sample images

(4) Replace the particles in situ at a different angle and take a picture again

(5) Use graphics software to sort out the particle images and extract 3D morphological parameters. The specific parameter determination method will be described in detail below

The size characteristics of the gravel particles are reflected by the aspect ratio, the short axis length, the central axis length, and the long axis length of particles. To be specific, the long axis length $L$ is the relatively large diameter in the $3 \mathrm{D}$ dimension of the particles, the central axis length $W$ is the maximum size of the vertical elevation with the long axis in the three-dimensional image of the particles, and the short axis length $t$ is the minimum diameter in the $3 \mathrm{D}$ diameter, which means $t$ is the longest diameter perpendicular to both the long axis and the central axis. The details are exhibited in Figure 3. The size ratio between the long axis and the short axis (aspect ratio, $L / t$ ) is selected to reflect the shape characteristics of the particles.

In the particle image, the angular characteristics of gravel particles are represented by the smoothness of the contour lines. As shown in Figure 4, on the contour line of the particle image, the perimeter of the contour line increases with the increase of the number of fluctuations. Based on this, a two-dimensional angular coefficient $\left(\mathrm{AC}_{2 \mathrm{D}}\right)$ is proposed to characterize the angularity of particles. The angular coefficient $\mathrm{AC}_{2 \mathrm{D}}$ is defined as the ratio of the difference in the surface contour circumference (SCC) and convex contour circumference (CCC) of the particles to the convex contour circumference (CCC) in the image, as shown in

$$
\mathrm{AC}_{2 \mathrm{D}}=\frac{\mathrm{SCC}-\mathrm{CCC}}{\mathrm{CCC}}
$$

in which SCC and CCC denote all the boundary lines of the particles themselves and the boundary line with high flatness that is obtained by ignoring the concave parts on the surface contour line, and the curve of the concave part of the particle surface is directly replaced by a straight line as the convex contour circumference.

In order to more realistically reflect the angular coefficient of a single particle, $\mathrm{AC}_{2 \mathrm{D}}$ of each side image is calculated, and the three-dimensional angular coefficient $\left(\mathrm{AC}_{3 \mathrm{D}}\right)$ of the particle is obtained by area weighted average, as shown in

$$
\mathrm{AC}_{3 \mathrm{D}}=\frac{\sum_{1}^{n} A_{i} \times \mathrm{AC}_{i}}{\sum_{1}^{n} A_{i}}
$$

where $n$ is the number of particles, $A_{i}$ is the area of the image $i$, and $\mathrm{AC}_{i}$ is the angular coefficient of the image $i$.

3.3. Results and Analysis. Figure 5 shows the frequency histograms and normal distribution $P-P$ graph of $L, W$, and $t$ for particle size gravel of $20 \sim 10 \mathrm{~mm}$. The horizontal axis and vertical axis of the frequency histogram of the 3D morphological parameters with different particle sizes represent the value interval and value frequency of the statistic, respectively. The $P-P$ graph is a statistical graph that detects the degree of conformity of distribution types. If the $P-P$ graph is displayed as a straight line from the origin of the vertical axis to the upper right, it means that the variable values follow a normal distribution [28]. In order to obtain the statistical distribution characteristics of $L / t$ and $\mathrm{AC}$, the $P-P$ graphs of Laplace, Logistic, lognormal, Pareto, Student $t$, Weibull, and uniform distribution of $L / t$ and $A C$ are explored, respectively. Figure 6 shows that $L / t$ and AC satisfy the lognormal distribution.

Using the same method, the probability statistics of the remaining two groups of gravel particles are performed, and the statistical parameter results are shown in Table 1. The three groups of gravel particles $L, W$, and $t$ 

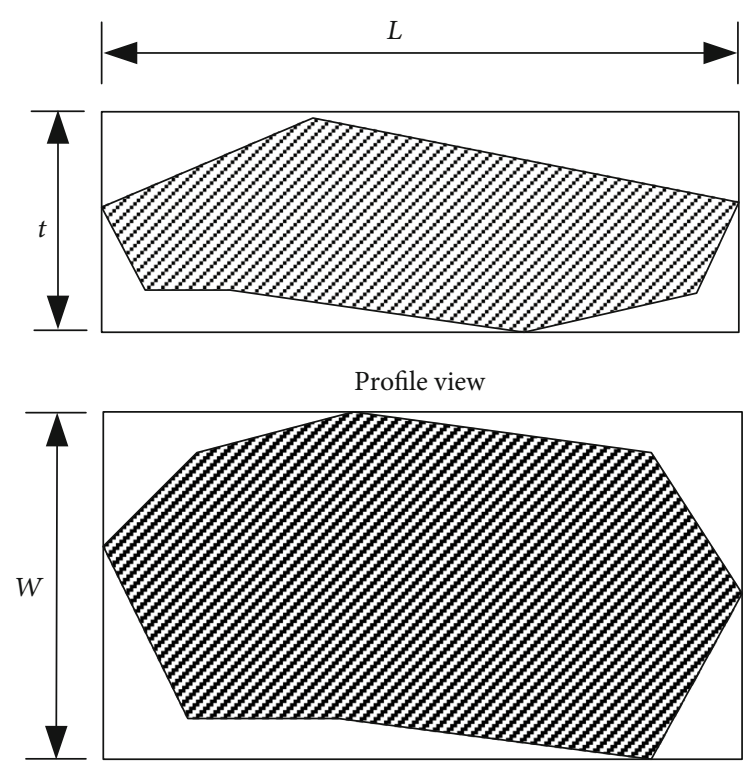

Plan view

Figure 3: Plan and profile views of the particles.

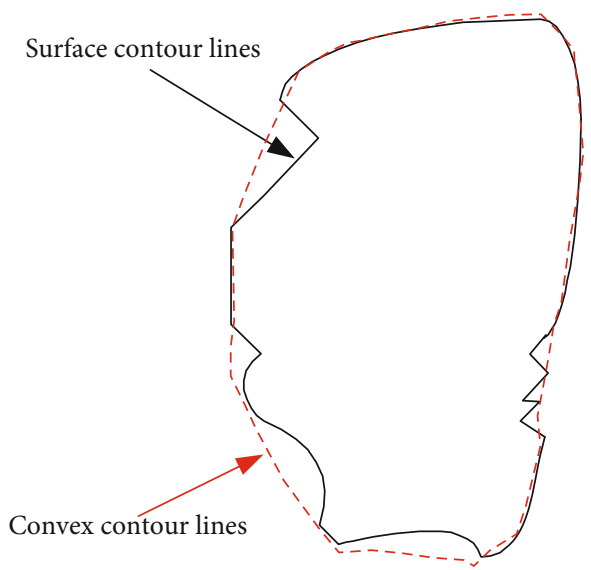

FIgURE 4: Surface contour lines and convex contour lines of the particle image.

approximately follow the normal distribution, while $L / t$ and $\mathrm{AC}$ approximately follow the lognormal distribution.

\section{The 3D Discrete Element Simulation of Sand- Gravel Mixtures}

4.1. Gravel Particle Simulation. The above research shows that the three-dimensional morphological distribution characteristics of gravel particles are obvious. Spherical particles, as the basic unit of the calculation model in PFC3D, differ greatly from the actual gravel particles. In this article, polyhedral particles are used as the basic unit to simulate the target material. Clump logic in the PFC3D software can generate super particles composed of a large number of spherical elements with diverse shapes and nondeform- able boundary conditions. At the same time, the effect of internal particles is ignored during calculation, so it can be regarded as a rigid whole for calculation. Zhang et al. [29] used clump super particles to simulate coarse aggregates based on PFC3D and proposed an algorithm for randomly generating the 3D morphology of coarse aggregate particles. The results showed that clump super particles can effectively make up the difference between traditional spherical particles and real materials. In order to improve the calculation accuracy and calculation efficiency, the author referred to reference $[30,31]$, focusing on the simulation of the $3 \mathrm{D}$ shape of gravel particles (greater than $5 \mathrm{~mm}$ ), using the equal-size particles with a diameter of $5 \mathrm{~mm}$ to simulate the sand particles, which reached the balance of accuracy and efficiency.

In order to better reflect the morphological characteristics of gravel particles, polyhedron (hexahedron, pentahedron, or tetrahedron) and angular coefficient were adopted to describe the $3 \mathrm{D}$ form of gravel particles. Hexahedron, pentahedron, or tetrahedron was used to reflect the main shape of particles. Meanwhile, the angular coefficient was introduced to describe the local morphological characteristics (smoothness of particle contour). As shown in Figure $7(\mathrm{a})$, the 3D shape of gravel particles was simulated approximately through hexahedron, pentahedron, or tetrahedron in the model. The 3D size of gravel particles was determined by the length of perpendicular edges $A B, A D$, and $A E$; meanwhile, the corner angle characteristic of particles was controlled by changing the angle of angle $A D H$, angle $A B F$, and angle $A B C$. Through the change of the above six parameters, the particles in different sizes and sharp edges could be generated. According to the statistical results, $L, W$, and $t$ of gravel particles with different particle sizes satisfied normal distribution, and $L / t$ and $A C$ satisfied logarithmic normal distribution. The mean value and standard deviation are shown in Table 2. According to the normal distribution, the model randomly selected the size parameters and angular parameters of particles to generate gravel grains with particle sizes of $50 \sim 20 \mathrm{~mm}, 20 \sim 10 \mathrm{~mm}$, and $10 \sim 5 \mathrm{~mm}$, as shown in Figure 7(b).

4.2. Sand Particle Simulation. The model simulated the sand particles with equal particle size with a minimum particle size of $5 \mathrm{~mm}$. The specific generation process is as follows:

(1) Calculate the number $N$ of spheres to be generated according to volume $V$, soil porosity $n$, and sphere radius $R$

(2) Use command "gen" in PFC3D to generate $N$ spheres with a radius of $0.2 r$ in the specimen, and assign the parameters of density and stiffness to the spheres. When the "gen" command is used to generate the spheres, the spheres are randomly generated within the sample and do not overlap each other, which ensures the uniformity of the sample

(3) Use command "mult" to enlarge the radius of the sphere by 5 times. There is a certain amount of overlap between the spheres 

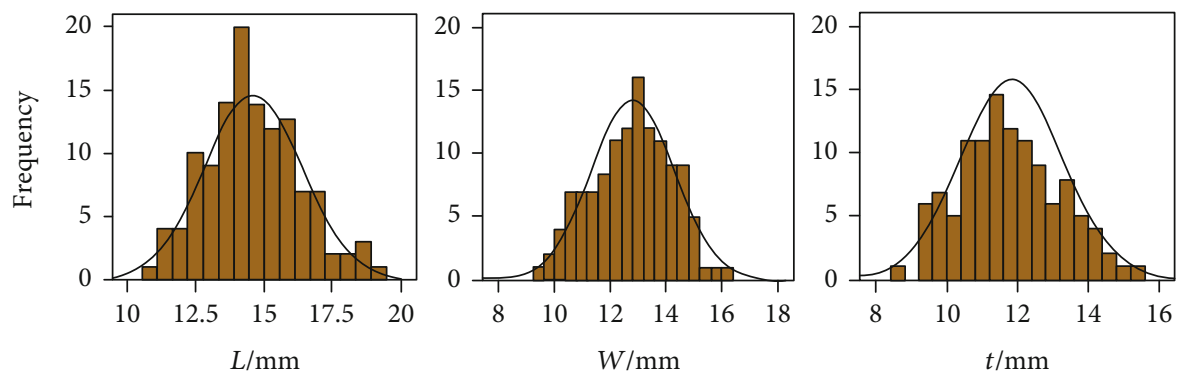

(a) Frequency histogram
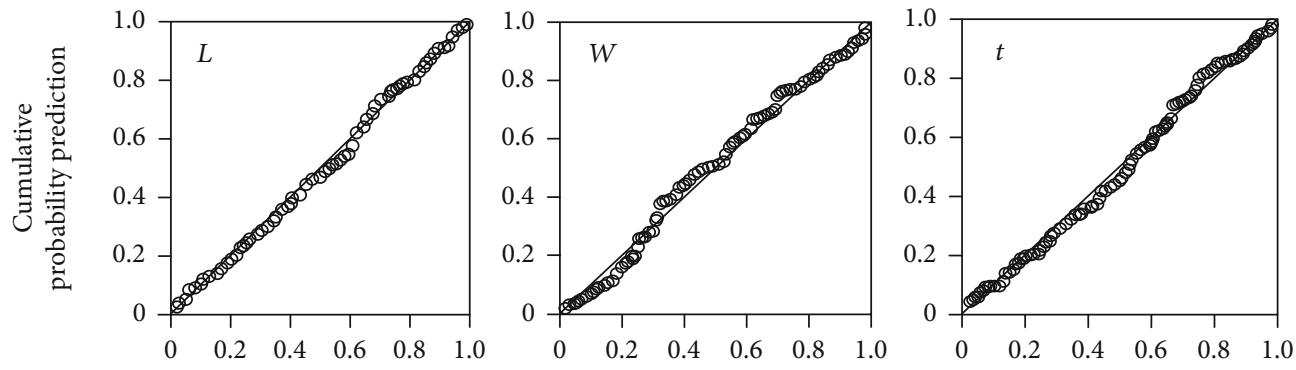

Cumulative probability observations

Cumulative probability observations

Cumulative probability observations

(b) Normal distribution $P$-P figure

Figure 5: Frequency histogram and normal distribution $P$-P figure of $L, W, t, L / t$, and AC.
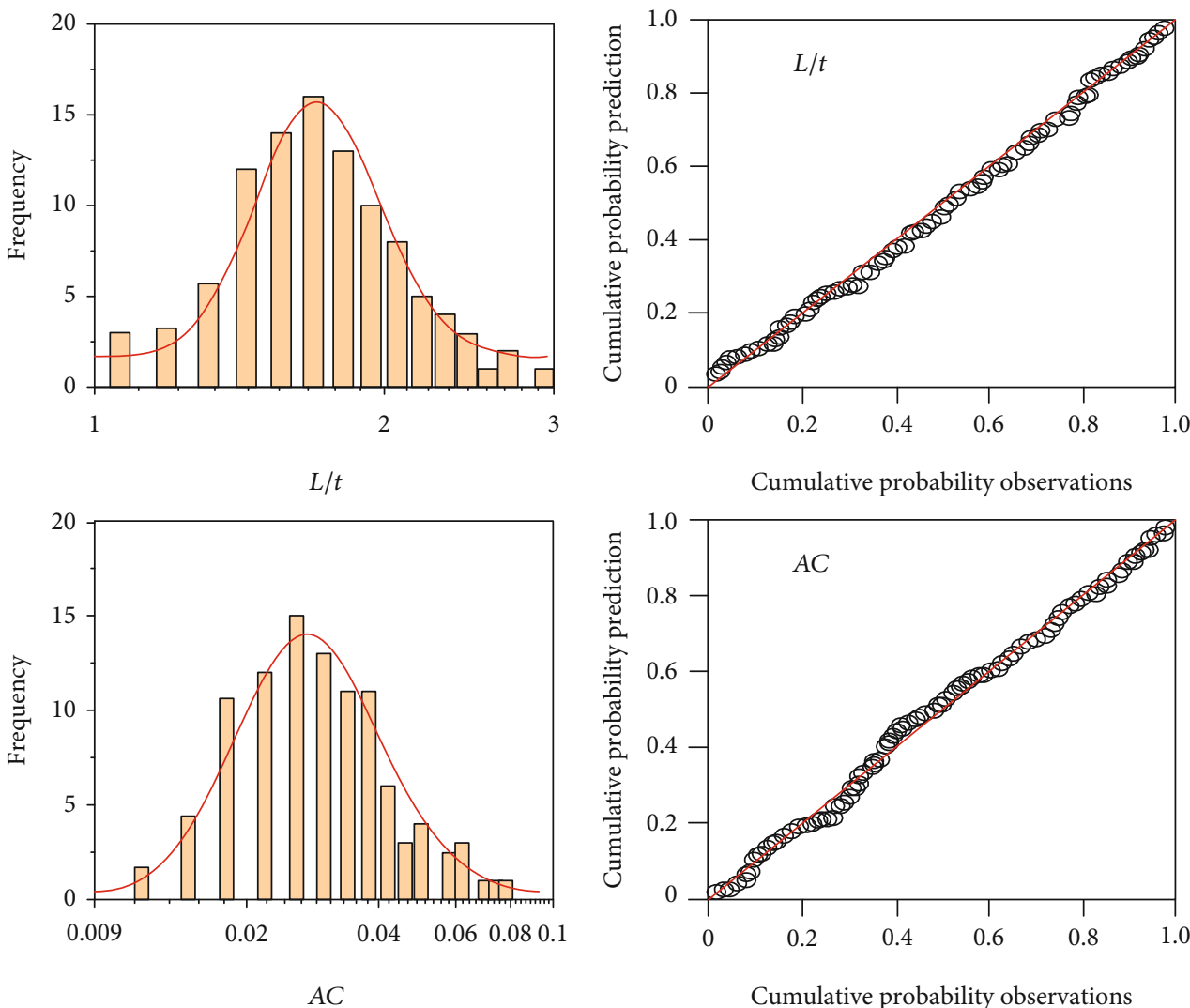

Figure 6: Frequency histogram and lognormal distribution $P$ - $P$ figures of $L / t$ and AC. 
TABLE 1: Statistical parameters of $L, W, t, L / t$, and $A$ for different particle size gravel.

\begin{tabular}{|c|c|c|c|c|c|c|c|c|c|c|}
\hline \multirow{2}{*}{$\begin{array}{l}d \\
(\mathrm{~mm})\end{array}$} & \multicolumn{2}{|c|}{$L(\mathrm{~mm})$} & \multicolumn{2}{|c|}{$W(\mathrm{~mm})$} & \multicolumn{2}{|c|}{$t(\mathrm{~mm})$} & \multicolumn{2}{|r|}{$L / t$} & \multicolumn{2}{|r|}{$\mathrm{AC}$} \\
\hline & Mean & $\begin{array}{l}\text { Standard } \\
\text { deviation }\end{array}$ & Mean & $\begin{array}{l}\text { Standard } \\
\text { deviation }\end{array}$ & Mean & $\begin{array}{l}\text { Standard } \\
\text { deviation }\end{array}$ & Mean & $\begin{array}{l}\text { Standard } \\
\text { deviation }\end{array}$ & Mean & $\begin{array}{l}\text { Standard } \\
\text { deviation }\end{array}$ \\
\hline $50 \sim 20$ & 43.12 & 10.16 & 30.34 & 7.75 & 25.53 & 6.81 & 1.72 & 0.30 & 0.026 & 0.011 \\
\hline $10 \sim 20$ & 23.78 & 5.33 & 17.02 & 3.70 & 14.55 & 3.63 & 1.69 & 0.39 & 0.030 & 0.013 \\
\hline $10 \sim 5$ & 12.14 & 2.35 & 8.70 & 1.70 & 7.82 & 1.63 & 1.59 & 0.32 & 0.019 & 0.007 \\
\hline
\end{tabular}

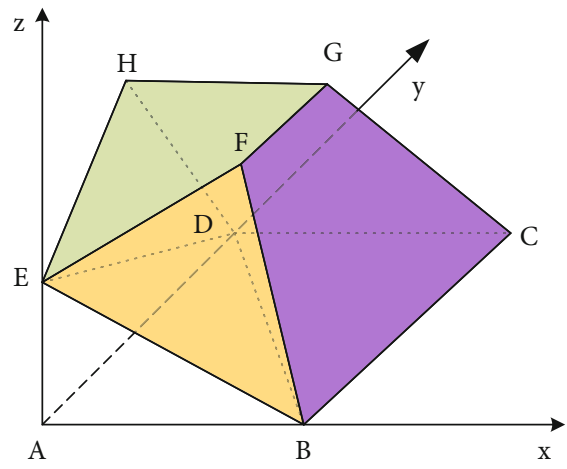

(a) Schematic drawing of the hexahedron

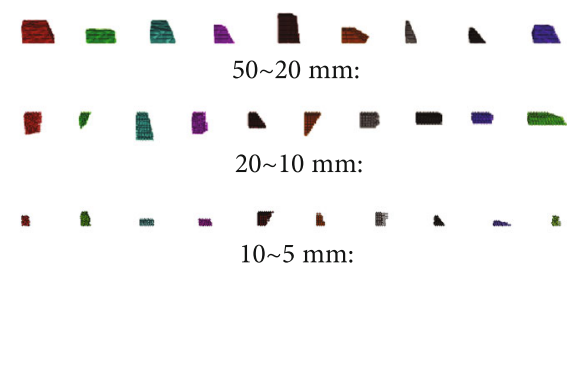

(b) Three-dimensional clump particle model

FIgURe 7: 3D clump particle model.

TABLE 2: Mesoparameters of particle for gravel-sand mixtures.

\begin{tabular}{lc}
\hline Parameter & Value \\
\hline Normal stiffness $(\mathrm{Pa})$ & $1 e 8$ \\
Tangential stiffness $(\mathrm{Pa})$ & $1 e 7$ \\
Coefficient of friction & 0.5 \\
Normal bond strength $(\mathrm{Pa})$ & 0 \\
Tangential bond strength $(\mathrm{Pa})$ & 0 \\
Unidirectional/cyclic shear rate & $001 \% / \mathrm{min}$ \\
Density $\left(\mathrm{g} / \mathrm{cm}^{3}\right)$ & 1709 \\
\hline
\end{tabular}

(4) Use command "cycle" to run the program to eliminate the overlap between the particles, so as to obtain a uniformly distributed sand-gravel soil

4.3. Triaxial Mesoscopic Simulation Test and Analysis. After the sample is generated, there will be overlap between the particles. The model will judge whether the sand particles and gravel particles overlap one by one. If the two coincide, delete the former. Figure 8 shows the discrete element samples of the triaxial test of the sand-gravel mixtures obtained by the above method. In order to simulate the loading process of the triaxial shear test, a square wall simulation loading indenter was generated at the upper and lower ends of the sample; a cylindrical wall used to simulate the confining pressure was generated on the side of the sample; during the test simulation, the wall at the upper and lower ends was controlled to apply an axial load to the sample at a certain speed, and the change in the radius of the side wall was controlled so that the surrounding pressure around the sample remained unchanged; the radial speed of the side wall was

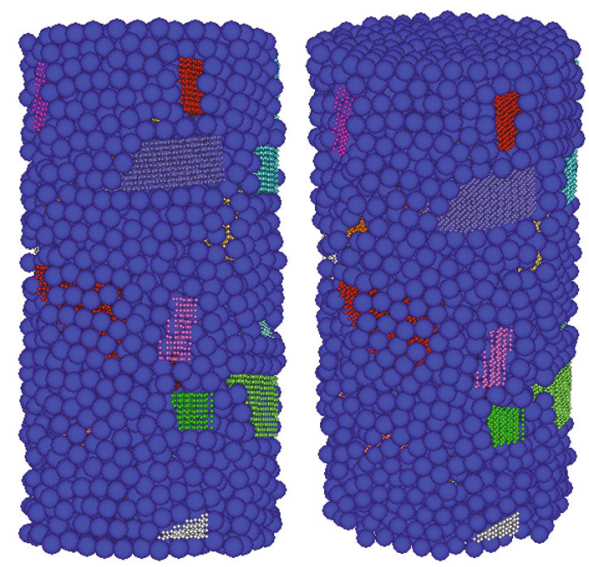

Figure 8: Discrete element sample of triaxial test.

automatically controlled using "numerical servomechanism." The method can realize the application of confining pressure and the loading of the test sample. Through repeated adjustments, the values of the mesoscopic parameters of sand-gravel mixtures are basically consistent with the test results. In this article, a set of ideal mesoscopic parameters of sand-gravel mixture models were calibrated, as shown in Table 2.

Figure 9 shows the drained shear test results and numerical simulation results of saturated sandy gravel mixtures when the initial effective confining pressure is $300 \mathrm{kPa}$. In the initial stage of loading, the deviatoric stress increases rapidly with the increase of the axial strain. As the axial strain further increases, the deviatoric stress slowly increases until the peak deviatoric stress is reached, and the volume 


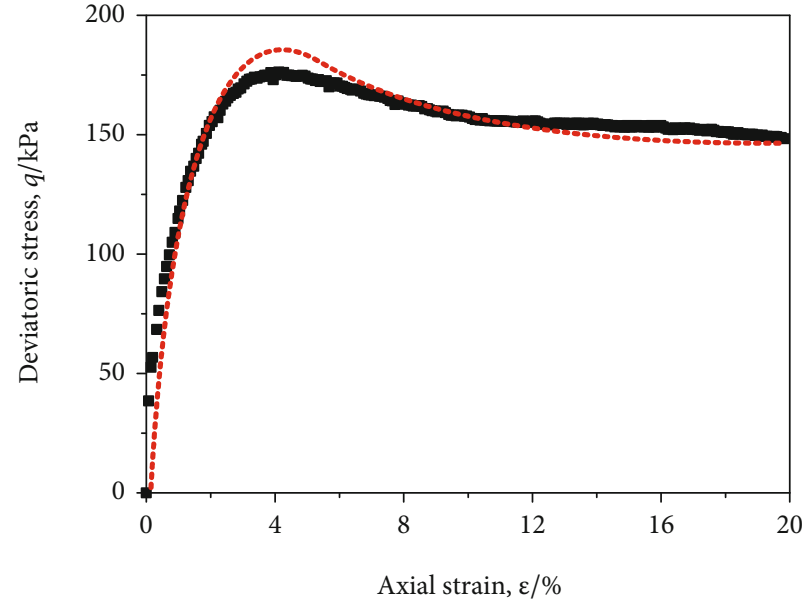

- Drained shear test ...... Numerical Simulation

(a)

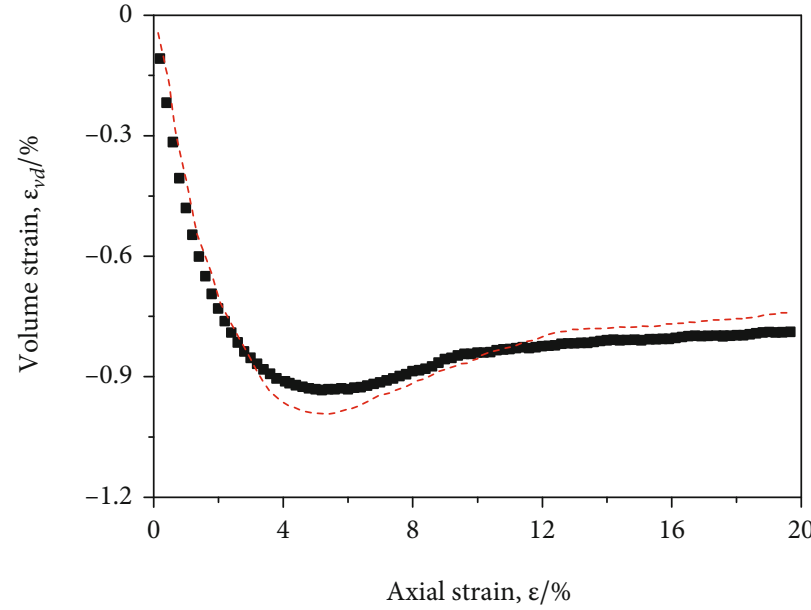

- Drained shear test Numerical Simulation

(b)

FIGURE 9: Deviatoric stress-axial strain and volume strain-axial strain curves of drained shear test.

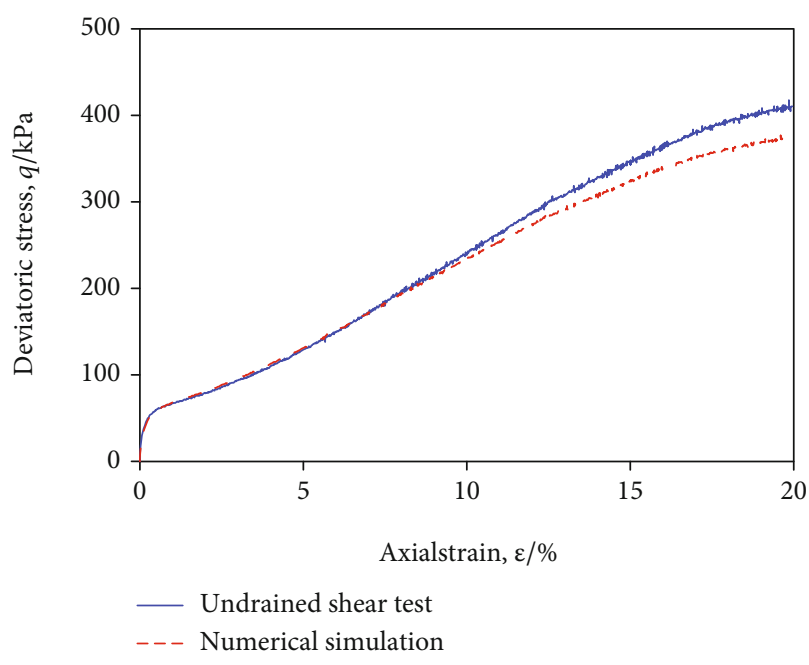

(a)

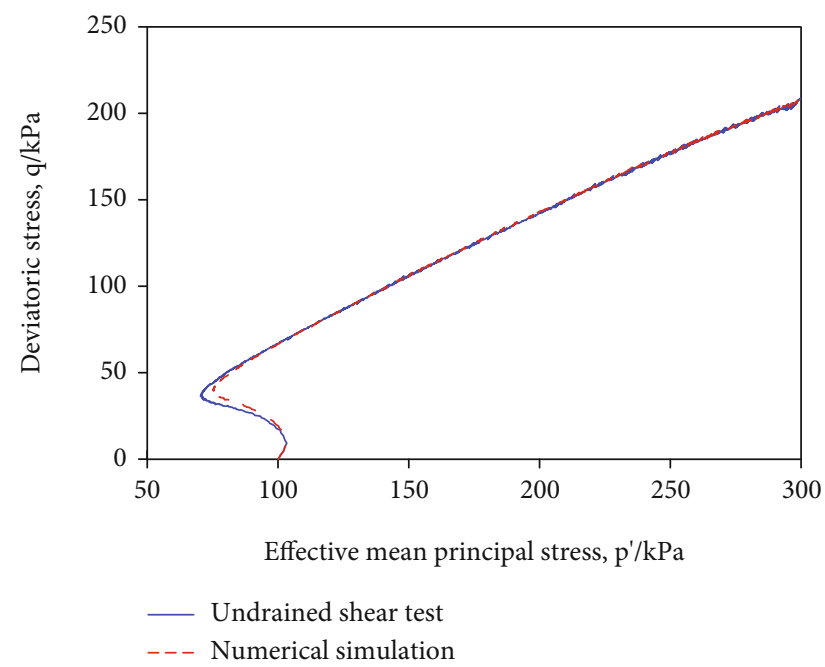

(b)

Figure 10: Deviatoric stress-axial strain curves and effective stress path of undrained shear test.

strain decreases with the increase of the axial strain; the soil appears to shrink. After that, the stress-strain relationship curve has obvious softening characteristics. As the axial strain increases, the deviatoric stress decreases. At this time, the axial strain-volume strain curve shows obvious shear expansion characteristics. It can be seen from Figure 9 that the numerical test results are in good agreement with the drained shear test results.

Figure 10 shows the undrained shear test results and numerical simulation results of saturated sandy gravel soil mixtures when the initial effective confining pressure is $300 \mathrm{kPa}$. It can be seen from the figure that when $\varepsilon<10 \%$, the difference between the numerical test results and undrained shear test results is very small. When $\varepsilon>10 \%$, the numerical test results is different from the undrained shear test results, and the difference increases with the increase of axial strain. This is because the sample is not completely stable when it is in large deformation, and the numerical simulation results cannot fully reflect the mechanical properties of the soil. It is worth noting that the effective stress path of the numerical test and the undrained shear test is basically the same.

Figure 11 shows the undrained cyclic triaxial test results and numerical simulation results of saturated sandy gravel mixtures when the initial effective confining pressure is $100 \mathrm{kPa}$. The pore pressure ratio $R_{\mathrm{u}}$ of gravel-sand mixtures presents a "fast-steady" growth pattern, which is basically consistent with the typical test results of gravel-sand mixtures. The stress-strain curve of the triaxial test and the numerical test can maintain good consistency before 


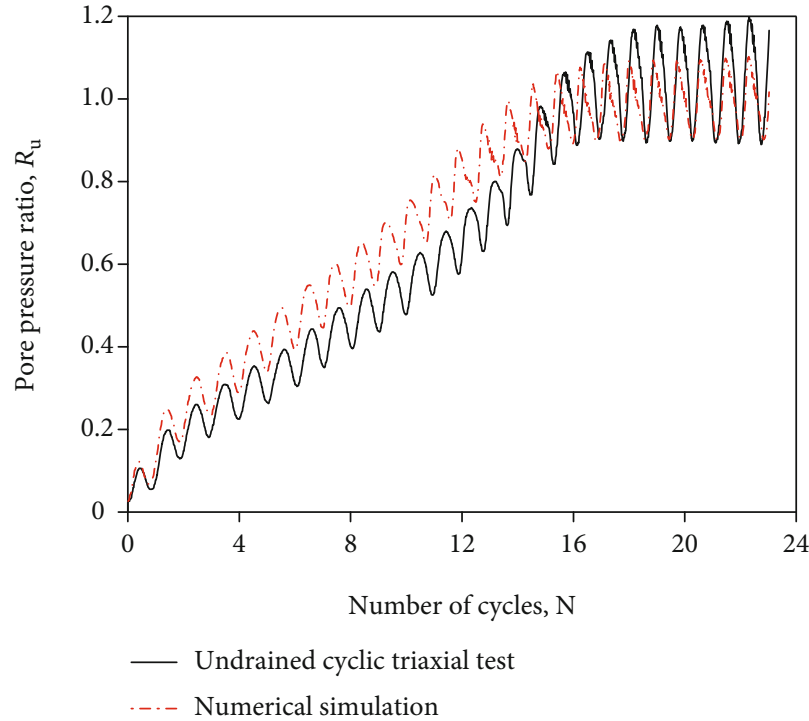

(a)

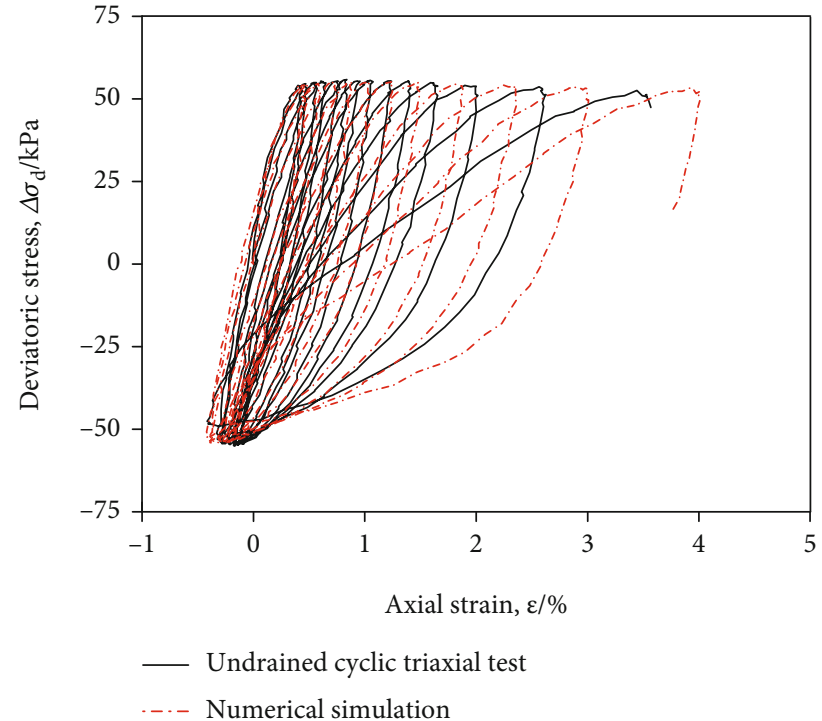

(b)

Figure 11: Pore pressure ratio-number of cycles and stress path of undrained cyclic triaxial test.

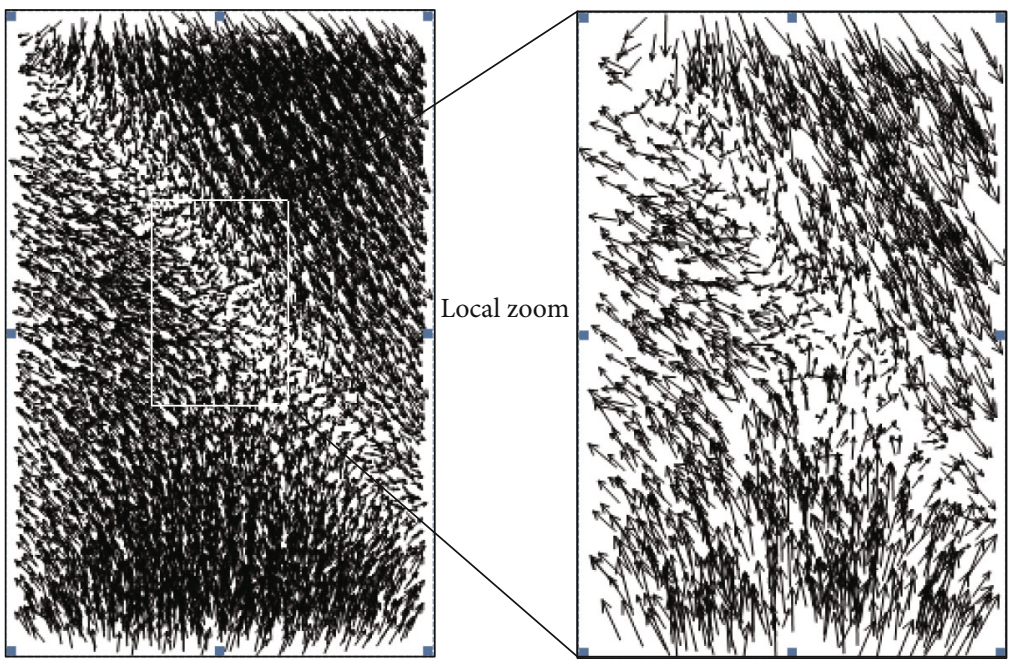

Figure 12: Distribution particle displacements.

liquefaction, and when the sample is liquefied, the sample no longer has shear strength. Meanwhile, it is difficult for the numerical test to accurately and effectively apply axial stress and reflect the true state of the sample, resulting in a certain difference in the stress-strain curve after liquefaction.

Because the particle flow sample is composed of discrete particles, similar to the actual coarse-grained soil, the interaction of the particles in the PFC3D numerical model can better reflect the mesomechanical properties of the actual sandy gravel mixtures. In the undrained shear numerical test, PFC3D provides the display of the force between particles. From the force between particles, the mesoscopic working mechanism can be analyzed. In order to study the particle movement law of the sandy gravel mixture sample, Figure 12 shows the particle displacement vector diagram of the numer- ical sample under shear failure when the initial effective confining pressure is $100 \mathrm{kPa}$. When shear failure occurs, the directional movement of the particles is obvious, the upper particles move downward and the lower particles move in the opposite direction, and obvious shear bands appear, indicating that structural damage occurs inside the sample.

Figures 13(a) and 13(b) show the distribution of sand particle contact force and gravel particle contact force, respectively. The soil composed of sand and gravel with obvious differences in particle size has a skeleton structure jointly undertaken by sand and gravel. However, in the case of this test, the distribution of the contact force between the sand particles is scattered, and the contact force between the gravel particles can form a complete contact force, so the gravel particles are mainly stressed particles. 


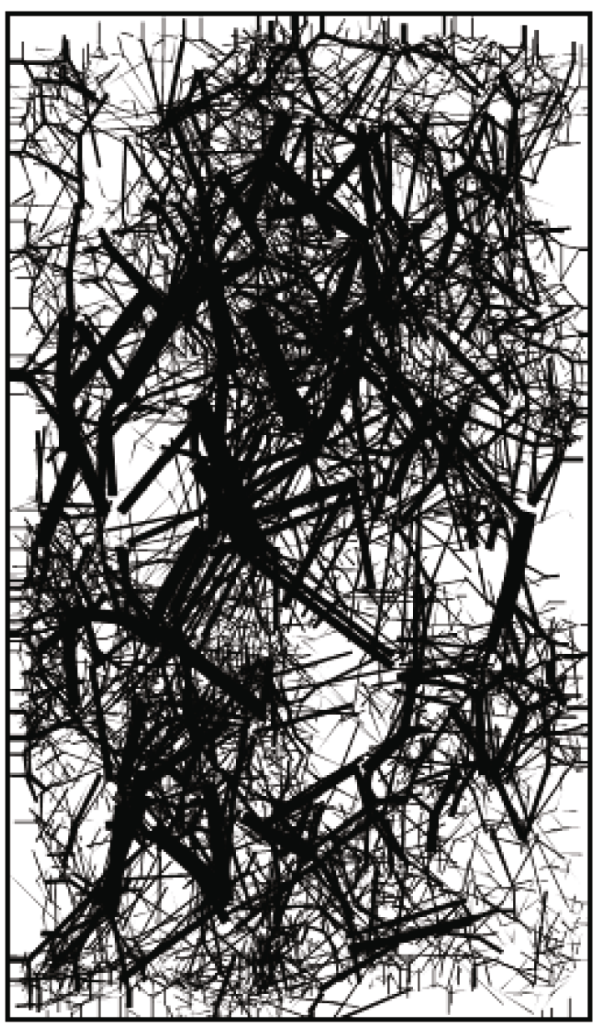

(a) Sand particle contact force diagram

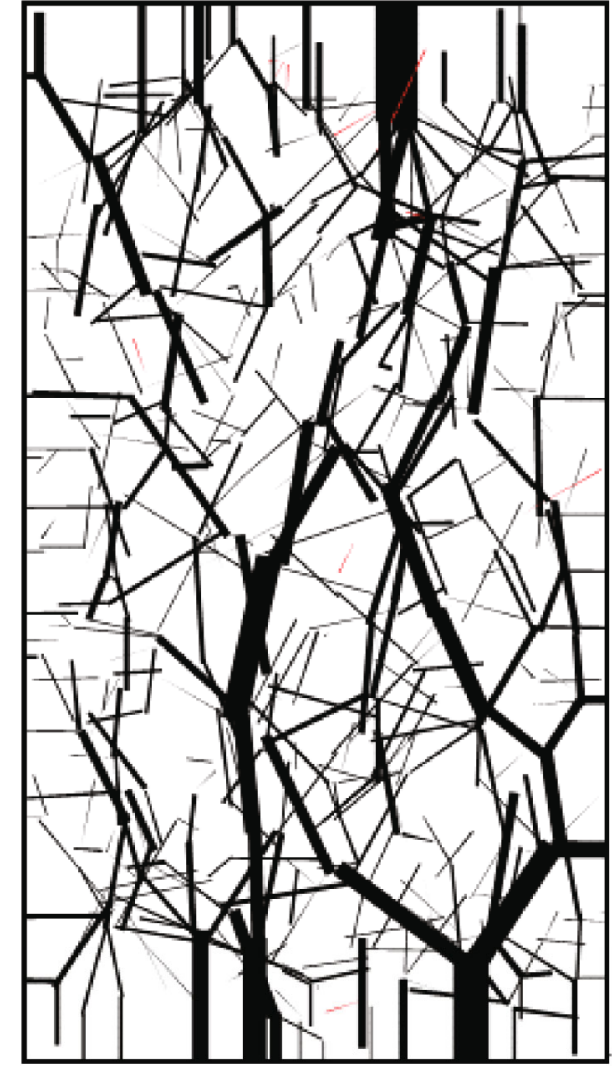

(b) Gravel particle contact force diagram

FIgURe 13: Particle contact force diagram.

The mechanical constitutive model of sand-gravel mixture established based on the 3D morphological distribution characteristics presents good applicability. The research results will provide an important scientific basis for the analysis of the stability and seismic performance of hydraulic fracturing drilling through crossing the sandy gravel mixture layer.

\section{Conclusions}

This article comprehensively used digital image processing technology and discrete element numerical simulation to study the mesoscopic structural characteristics and the PFC3D particle flow simulation method of the sandgravel mixtures taken from the Chengdu metro area in China. According to the statistical characteristics of the 3D form and distribution of the particles, a 3D discrete element simulation method for sandy gravel mixtures is proposed. The discrete element method was used to analyze the particle movement law of the sand-gravel mixtures during the shear test process. The main conclusions are as follows:

(1) The long axis length $L$, the middle axis length $W$, and the short axis length $t$ of the gravel particles in accordance with the normal distribution and the aspect ratio $L / t$ and the angular coefficient $A C$ satisfy the lognormal distribution. The PFC3D particle flow model can well simulate the 3D morphological distribution characteristics of gravel particles to form uniformly distributed sand-gravel mixtures

(2) The deviatoric stress-axial strain curves obtained from the simulated drained shear test, undrained shear test, and undrained cyclic triaxial test are in good agreement with the test results, which verifies the applicability of the model

(3) From the particle contact force diagram between the particles, it is concluded that the gravel particles in the sand-gravel mixtures are forced particles during the drained shear process

\section{Data Availability}

The data are generated from experiments and can be available from the corresponding author upon request.

\section{Conflicts of Interest}

The authors declare that there are no conflicts of interest regarding the publication of this paper. 


\section{Acknowledgments}

This work was supported by the National Science Foundation of China (52008206 and 51978334) and Institute of Crustal Dynamics, China Earthquake Administration (No. ZDJ2018-11).

\section{References}

[1] L. Chen, Z. Wang, X. Peng, J. Yang, P. Wu, and H. Lian, "Modeling pressurized fracture propagation with the isogeometric BEM," Geomechanics and Geophysics for Geo-Energy and Geo-Resources, vol. 7, no. 3, 2021.

[2] J. F. Yang, H. Lian, and L. Li, "Fracturing in coals with different fluids: an experimental comparison between water, liquid $\mathrm{CO}_{2}$, and supercritical $\mathrm{CO}_{2}$," Scientific Reports, vol. 10, no. 1, 2020.

[3] Z. H. Liu, J. Yang, L. S. Yang, X. Ren, X. Peng, and H. Lian, "Experimental study on the influencing factors of hydraulic fracture initiation from prefabricated crack tips," Engineering Fracture Mechanics, vol. 250, 2021.

[4] Z. H. Liu, X. K. Ren, X. Lin, H. Lian, L. Yang, and J. Yang, "Effects of confining stresses, pre-crack inclination angles and injection rates: observations from large-scale true triaxial and hydraulic fracturing tests in laboratory," Rock Mechanics and Rock Engineering, vol. 53, no. 4, pp. 1991-2000, 2020.

[5] H. Zhuang, R. Wang, G. Chen, Y. Miao, and K. Zhao, "Shear modulus reduction of saturated sand under large liquefaction-induced deformation in cyclic torsional shear tests," Engineering Geology, vol. 240, pp. 110-122, 2018.

[6] B. Ruan, K. Zhao, S. Y. Wang, G. X. Chen, and H. Y. Wang, "Numerical modeling of seismic site effects in a shallow estuarine bay (Suai Bay, Shantou, China)," Engineering Geology, vol. 260, 2019.

[7] M. D. Evans and S. Zhou, "Liquefaction behavior of sandgravel composites," Journal of Geotechnical and Geoenvironmental Engineering, vol. 121, no. 3, pp. 287-298, 1995.

[8] Y. R. Li, R. Q. Huang, L. S. Chan, and J. Chen, "Effects of particle shape on shear strength of clay-gravel mixture," KSCE Journal of Civil Engineering, vol. 17, no. 4, pp. 712-717, 2013.

[9] T. D. O'Rourke, S. J. Druschel, and A. N. Netravali, "Shear strength characteristics of sand-polymer interfaces," Journal of Geotechnical Engineering, vol. 116, no. 3, pp. 451-469, 1990.

[10] L. E. Vallejo, "Interpretation of the limits in shear strength in binary granular mixtures," Canadian Geotechnical Journal, vol. 38, no. 5, pp. 1097-1104, 2011.

[11] W. J. Chang, "Evaluation of liquefaction resistance for gravelly sands using gravel content-corrected shear-wave velocity," Journal of Geotechnical \& Geoenvironmental Engineering, vol. 142, no. 5, 2016.

[12] K. Zhao, Q. Z. Wang, Q. Chen, H. Zhuang, and G. Chen, "Simplified effective stress simulation of shear wave propagation in saturated granular soils," Géotechnique Letters, vol. 11, no. 1, pp. 1-22, 2021.

[13] S. Zhang, H. M. Tang, H. B. Zhan, G. Lei, and H. Cheng, "Investigation of scale effect of numerical unconfined compression strengths of virtual colluvial-deluvial soil-rock mixture," International Journal of Rock Mechanics and Mining Sciences, vol. 77, pp. 208-219, 2015.

[14] S. K. Kennedy and W. H. Lin, "A comparison of fourier and fractal techniques in the analysis of closed forms," Journal of Sedimentary Research, vol. 62, no. 5, pp. 842-848, 1992.
[15] E. T. Bowman, K. Soga, and W. Drummond, "Particle shape characterisation using Fourier descriptor analysis," Géotechnique, vol. 51, no. 6, pp. 545-554, 2001.

[16] B. Sukumaran and A. K. Ashmawy, "Quantitative characterisation of the geometry of discret particles," Géotechnique, vol. 51, no. 7, pp. 619-627, 2001.

[17] G. C. Cho, J. Dodds, and J. C. Santamarina, "Closure to 'particle shape effects on packing density, stiffness, and strength: natural and crushed sands' by Gye-Chun Cho, Jake Dodds, and J. Carlos Santamarina," Journal of Geotechnical and Geoenvironmental Engineering, vol. 133, no. 11, pp. 1474-1474, 2007.

[18] J. F. Ferellec and G. R. Mcdowell, "A simple method to create complex particle shapes for dem," Geomechanics and Geoengineering, vol. 3, no. 3, pp. 211-216, 2008.

[19] A. Cividini and G. Gioda, "A finite element analysis of direct shear tests on stiff clays," International Journal for Numerical \& Analytical Methods in Geomechanics, vol. 16, no. 12, pp. 869-886, 1992.

[20] J. Tejchman, "FE analysis of shearing of granular bodies in a direct shear box," Particulate Science and Technology, vol. 23, no. 3, pp. 229-248, 2005.

[21] P. Mora and D. Place, "A lattice solid model for the nonlinear dynamics of earthquakes," International Journal of Modern Physics C, vol. 4, no. 6, pp. 1059-1074, 1993.

[22] G. R. Mcdowell and O. Harireche, "Discrete element modelling of soil particle fracture," Géotechnique, vol. 52, no. 2, pp. 131-135, 2002.

[23] Y. P. Cheng, Y. Nakata, and M. D. Bolton, "Discrete element simulation of crushable soil," Geotechnique, vol. 53, no. 7, pp. 633-641, 2003.

[24] R. Deluzarche and B. Cambou, "Discrete numerical modelling of rockfill dams," International Journal for Numerical \& Analytical Methods in Geomechanics, vol. 30, no. 11, pp. 1075-1096, 2006.

[25] E. Alaei and A. Mahboubi, "A discrete model for simulating shear strength and deformation behaviour of rockfill material, considering the particle breakage phenomenon," Granular Matter, vol. 14, no. 6, pp. 707-717, 2012.

[26] E. E. Alonso, M. Tapias, and J. Gili, "Scale effects in rockfill behaviour," Géotechnique Letters, vol. 2, no. 3, pp. 155-160, 2012.

[27] ASTM, "Standard practice for classification of soils for engineering purposes (unified soil classification system)," in ASTM D2487, ASTM, West Conshohocken, PA, 2011.

[28] W. J. Xu, Z. Q. Yue, and R. L. Hu, "Study on the mesostructure and mesomechanical characteristics of the soil-rock mixture using digital image processing based finite element method," International Journal of Rock Mechanics \& Mining Sciences, vol. 45, no. 5, pp. 749-762, 2008.

[29] D. Zhang, X. M. Huang, and Y. L. Zhao, "Algorithms for generating three-dimensional aggregates and asphalt mixture samples by the discrete-element method," Journal of Computing in Civil Engineering, vol. 27, no. 2, pp. 111-117, 2013.

[30] B. W. Flemming, "A revised textural classification of gravelfree muddy sediments on the basis of ternary diagrams," Continental Shelf Research, vol. 20, no. 10-11, pp. 1125-1137, 2000.

[31] I. López, L. Aragonés, Y. Villacampa, P. Compañ, and R. Satorre, "Morphological classification of microtidal sand and gravel beaches," Ocean Engineering, vol. 109, pp. 309$319,2015$. 